\title{
PCR-RFLP method enhance DNA sequencing of IDH1 somatic mutations detection in gliomas
}

\author{
Wei Chiang Goh 1, Badrisyah Idris ', Regunath Kandasamy 1, Shaharum Shamsuddin 2, Hasnan Jaafar ${ }^{3}$, \\ Farizan Ahmad ${ }^{1}$ \\ (1) Universiti Sains Malaysia, Department of Neuroscience, School of Medical Science, Kubang Kerian, Malaysia \\ (2) Universiti Sains Malaysia, School of Health Science, Kubang Kerian, Malaysia \\ (3) Universiti Sains Malaysia, Department of Pathology, School of Medical Science, Kubang Kerian, Malaysia
}

Date submitted:

Jun 24, 2019

Date accepted:

Sep 11, 2019

Online publication date:

December 15, 2019

\section{Corresponding Author: \\ Farizan Ahmad \\ Universiti Sains Malaysia, \\ Department of Neuroscience, \\ Kubang Kerian, Malaysia \\ farizan@usm.my}

Keywords: Glioma, IDH1 R132L, IDH1 R132H, DNA sequencing, PCR-RFLP.

\section{ABSTRACT}

Aims:Isocitrate dehydrogenase 1 (IDH1) mutations are commonly found in lower grade gliomas and secondary GBMs. Glioblastomas (GBMs) are the most common and most malignant gliomas in adult with a median survival of 15 months only. Discovery of IDH1 mutations have made a significant positive impact on glioma patient's diagnosis, prognosis, and treatments. Thus, IDH1 mutation screening method in gliomas is necessary to improve survival rate. The gold standard for somatic mutation screening, DNA sequencing was performed to detect IDH1 gene mutation status in glioma samples.

Methods:Forty-seven formalin fixed paraffin-embedded glioma samples were subjected for DNA sequencing. The ambiguous glioma samples from DNA sequencing were subjected to PCR-RFLP for IDH1 mutation confirmation.

Results:Three out of 47 glioma samples (6.4\%) were found to harbor IDH1 mutations. Two IDH1 R132H and one IDH1 R132L were found in the glioma samples. From the DNA sequencing results, we found that the mutant nucleotide spectrum was lower than the wild-type nucleotide results in failure of IDH1 mutations detection. PCR-RFLP method was implemented to confirm the ambiguous IDH1 mutations. We found that the ambiguous IDH1 mutations from DNA sequencing were indeed IDH1 mutants using PCR-RFLP method.

Conclusions:In conclusion, DNA sequencing method has a considerable low sensitivity level which leads to false negative results. Thus, combination of DNA sequencing and PCR-RFLP method in heterogeneous glioma samples can be applied to avoid false negative result and cost-effective.

\section{Introduction}

Gliomas are the most frequent primary brain tumors in adults and categorized into four grades, grade I, II, III and IV (1). Annually, approximately 20,000 people in the United State suffered from gliomas (2). Globocan 2012 predicted that the annual incident of central nervous system (CNS) related tumors in Malaysia to be 2.8 in every 100,000 population with a cumulative rate of $0.3 \%(3)$.

Isocitrate Dehydrogenase 1 (IDH1) gene is located at the chromosome 2q33.3 and present in cytoplasm and peroxisome (4). IDH1 enzyme catalyzes oxidative decarboxylation of isocitrate to $\alpha$-ketoglutarate $(\alpha-K G)$ and carbon dioxide $(\mathrm{CO} 2)$ in Krebs cycle. IDH1 somatic mutations were discovered in $12 \%$ of GBM patients at codon R132 during exome-wide sequencing (5). IDH1 mutant enzyme interacts with IDH1 wild-type enzyme to inhibit IDH1 wild-type activity (6). IDH1 mutant enzyme also exhibits new enzymatic function that catalyzes conversion of a-ketoglutarate to D-2-hydroxyglutarate (D-2HG) (7).

IDH1 mutations showed high diagnostic value in gliomas as it was common in lower grade gliomas and secondary GBMs but rare in primary GBMs (8). It is important to determine glioma patients prognosis as IDH1 mutant gliomas showed favorable prognosis compared to the IDH1 wild-type gliomas (9). IDH1 mutations have high therapeutic values for development of targeted IDH1 mutants gliomas (10).

DNA sequencing is the gold standard for somatic mutation detection in clinical settings (11). DNA sequencing has been applied in various gliomas screening (12-14). So far, only one study was performed in Malaysia specifically targeting IDH1 $\mathrm{R} 132 \mathrm{H}$ mutation in brain tumors (15). The aim of our study was to determine other possible IDH1 mutations present in the gliomas via DNA sequencing method.

Here, we have performed DNA sequencing and PCR-RFLP methods for IDH1 mutation analysis in 47 glioma samples consisting of different gliomas types and grades from Hospital Universiti Sains Malaysia, Malaysia.

\section{Methods}

\section{Tumor samples}

A total of 47 archived formalin-fixed paraffin embedded 
(FFPE) glioma specimens were obtained from Pathology Department, Hospital Universiti Sains Malaysia. The FFPE collection was performed with ethical approval (Ref. no. USM/ JEPeM/17050255) from Human Research Ethics Committee of Universiti Sains Malaysia (JePem). A pathologist reviewed the FFPE glioma blocks for gliomas confirmation according to latest World Health Organization (WHO) criteria (16) and assessment of neoplastic cellularity. Only FFPE glioma blocks with $\geq 40 \%$ tumor cells were chosen for this study. This study included 2 grade I pilocytic astrocytomas, 7 grade II astrocytomas, 9 grade III anaplastic astrocytomas, 25 grade IV GBMs, 1 grade II oligodendroglioma, 1 grade III anaplastic oligodendroglioma, 1 grade III anaplastic oligoastrocytoma and 1 grade III anaplastic ependymomas.

\section{DNA extraction}

Genomic DNA was extracted from the FFPE glioma blocks using Exgene Cell SV mini (GeneAll, Republic of Korea) according to the manufacturer's instructions. The concentration and purity of the genomic DNA were determined using NanoDrop 2000 Spectrophotometer (Thermo Fisher Scientific, USA) and stored at $-20^{\circ} \mathrm{C}$.

\section{Polymerase Chain Reaction (PCR) and DNA sequencing}

PCR primer sequences, 5'-AATGAGCTCTATATGCCATCACTG-3' (forward) and 5'-TTCATACCTTGCTTAATGGGTGT-3' (reverse) were used to amplify 500bp IDH1 PCR amplicon (4) using KOD Hot Start DNA Polymerase (Toyobo, Japan). PCR amplification was performed in a total of $50 \mu \mathrm{L}$ reaction containing 100ng genomic DNA, $5 \mu \mathrm{L}$ of 10X PCR buffer, $3 \mu \mathrm{L}$ of $25 \mathrm{mM} \mathrm{MgSO} 4,5 \mu \mathrm{L}$ of $2 \mathrm{mM}$ dNTP each, $1 \mu \mathrm{L}$ of $10 \mu \mathrm{M}$ forward and reverse primers and $1 \mu \mathrm{L}$ of KOD Hot Start DNA Polymerase enzyme. Thermocycling conditions were $95^{\circ} \mathrm{C}$ for 2 minutes, followed by 35 cycles of $95^{\circ} \mathrm{C}$ for 20 seconds, annealing for $10 \mathrm{~s}$ at $53^{\circ} \mathrm{C}$ and extension at $70^{\circ} \mathrm{C}$ for $10 \mathrm{~s}$, and finally $70^{\circ} \mathrm{C}$ for $5 \mathrm{~min}$. PCR products were analyzed using $2 \%$ agarose gel. After confirmation of expected 500bp PCR amplicon size, the PCR products were sent for sequencing using the same primer by First BASE Laboratory, Malaysia. The DNA sequences were aligned with IDH1 wild-type sequences from NCBI database (NM_005896.2) using NCBI BLAST software and electropherogram analysis using the Sequence Scanner software 2 version 2.0 (Applied Biosystem, USA).

\section{Restriction Fragment Length Polymorphism, PCR-RFLP}

A set of IDH1 mismatch primer, 5'-TGGGTAAAACCTATCATCATCGAT-3'(forward) and 5'-TGTGTTGAGATGGACGCCTA-3'(reverse) was used to introduce Pvul restriction site at the codon 132 (17). PCR amplification was performed in a total of $50 \mu \mathrm{L}$ PCR mixture containing $5 \mu \mathrm{L}$ of previously amplified $500 \mathrm{bp}$ PCR product, $5 \mu \mathrm{L}$ of $10 \mathrm{X} \mathrm{NH} 4$ buffer, $3 \mu \mathrm{L}$ of $50 \mathrm{mM}$ $\mathrm{MgCl} 2,0.5 \mu \mathrm{L}$ of $100 \mathrm{mM}$ dNTP mix, $1 \mu \mathrm{L}$ of $10 \mu \mathrm{M}$ forward and reverse primers and $1 \mu \mathrm{L}$ of BioTaq DNA Polymerase enzyme (Bioline, UK). Thermocycling conditions were $95^{\circ} \mathrm{C}$ for 5 minutes, followed by 35 cycles of $95^{\circ} \mathrm{C}$ for 30 seconds, annealing at $56^{\circ} \mathrm{C}$ for 30 seconds, extension at $72^{\circ} \mathrm{C}$ for 15 seconds and finally $72^{\circ} \mathrm{C}$ for 5 minutes. The PCR products were analyzed using $2 \%$ agarose gel to confirm the expected PCR amplicon (261bp). The digestion mixture was as set up as follow, $2 \mu \mathrm{L}$ of 10X CutSmart buffer, 10 $\mu \mathrm{L}$ PCR product, $1 \mu \mathrm{L}$ of Pvul-HF restriction enzyme (New England Biolab, USA) incubated at $37^{\circ} \mathrm{C}$ for 1 hour. The digested PCR products were subjected to $4 \%$ agarose gel electrophoresis at 80 Volts for 90 minutes.

\section{Analysis}

The association of IDH1 gene status with the clinicopathological parameters (age, gender, and histological subtypes) of the patients were analyzed via descriptive analysis.

\section{Results}

A total of 47 glioma samples were subjected to PCR amplification. Figure 1 showed the results of PCR amplification of IDH1 gene consisting codon 132 from the glioma samples. PCR products were sequenced and the electropherogram results were showed in Figure 2.

From our IDH1 analysis using DNA sequencing method, we
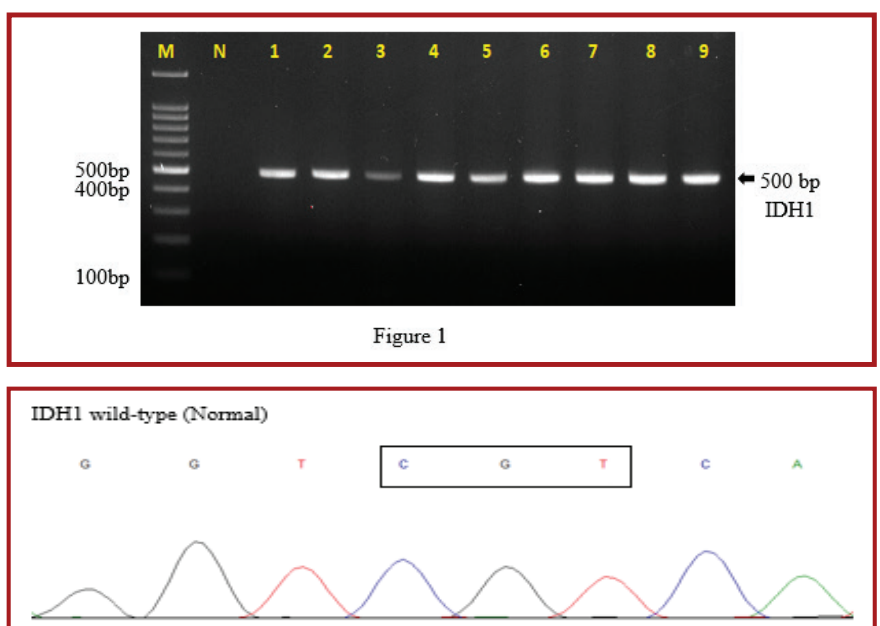

IDHI R132H (c.395G>A)

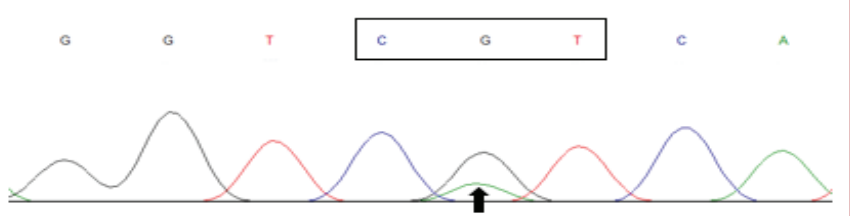

IDH1 R132L (c.395G > T)
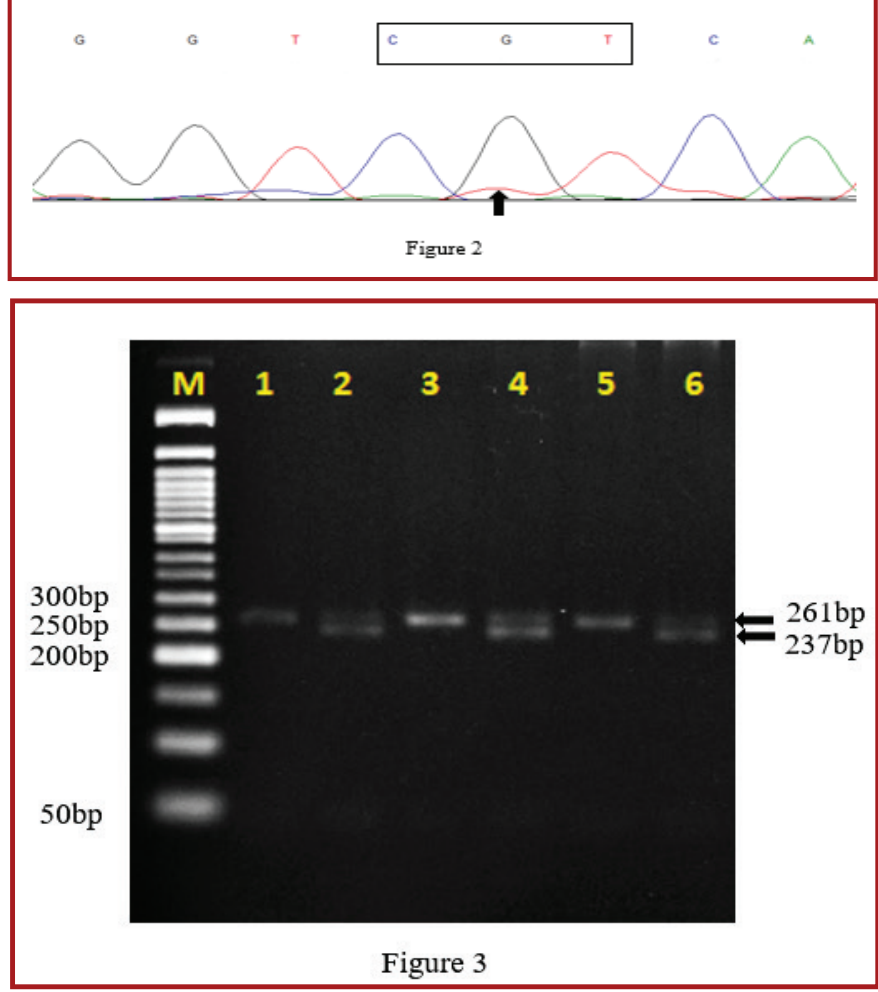
were unable to find IDH1 mutations. However, the presence of smaller peak spectrum at the position 395th nucleotide from the electropherogram results make the IDH1 gene status ambiguous (Figure 2). Therefore, three ambiguous glioma samples were subjected to PCR-RFLP method to confirm the IDH1 gene (Figure 3). The amplified PCR amplicon (261bp) was subjected to Pvul digestion. After digestion, gliomas with IDH1 heterozygous mutation showed 2 PCR fragments which were $261 \mathrm{bp}$ (IDH1 mutant) and 237bp (IDH1 wild-type).

Due to the presence of two DNA fragments from PCR-RFLP and DNA electropherograms, we confirmed that the three glioma samples were indeed harboring IDH1 R132H and IDH1 R132L mutations. Among the 47 glioma samples, two IDH1 $\mathrm{R} 132 \mathrm{H}$ and one IDH1 R132L were discovered. The association of IDH1 gene status with clinical characteristic of patients (tumor types and grades) and baseline characteristic of patients (age, gender and race) were showed in Table 1 and Table 2 respectively.

\section{Discussion}

DNA analysis is more preferable compared to the immunohistochemistry as it gives more objective interpretation of the genetic status. Since IDH1 mutations have high diagnostic value in gliomas, it can be used to reduce the subjective interpretation by different neuropathologists (9).

Immunohistochemistry have few drawbacks in IDH1 muta- tions detection. Anti-IDH1 R132H antibody was used to detect the most common IDH1 mutation, IDH1 R132H in the glioma samples (18). However, other IDH1 mutations cannot be detected by such method and have be confirmed via DNA analysis. Immunostaining often shows non-specific background staining or regional heterogeneity of IDH1-R132H protein expression which undermine the tumor's true malignancy. The IDH1-R132H antibody was found to cross-react with IDH1 wildtype and other IDH1 mutations, IDH1 R132L that leads to false interpretations (4).

IDH1 hotspot mutations were widely known to occur at codon R132 involving 394th, 395th and 396th nucleotides (19). From the DNA fasta sequencing results, we were unable to find mutant nucleotides but we did discovered there was another smaller peak spectrum at 395th nucleotide, Guanine $(G)$ based on the DNA electropherogram results. At this point, we cannot confirm whether the presence of smaller nucleotide peak is due to the sequencing background noise or actual IDH1 mutation. Therefore, we opted PCR-RFLP method (17) to confirm the ambiguous samples. This method converts the IDH1 wild-type nucleotide sequences 5'AGGTCG3' to become 5'CGATCG3', Pvul restriction site where CG at the $3^{\prime}$ end is part of IDH1 hotspot mutation site. After Pvul digestion, the presence of two bands indicated two different IDH1 genes in the samples, IDH1 wild-type and IDH1 mutant (Figure 3). Therefore, the presence of IDH1 mutation in the glioma samples were confirmed.

\section{Table 1: Association between the IDH1 gene status with tumor types and grades among 47 glioma samples.}

\begin{tabular}{|c|c|c|c|c|}
\hline \multirow[b]{2}{*}{ Characteristic } & \multirow[b]{2}{*}{ No. of samples } & \multicolumn{3}{|c|}{ IDH1 status } \\
\hline & & IDH1 R132H & IDH1 R132L & $\begin{array}{c}\text { IDH1 } \\
\text { wild-type }\end{array}$ \\
\hline No of samples & 47 & $2(4.3 \%)$ & $1(2.1 \%)$ & $44(93.6 \%)$ \\
\hline \multicolumn{5}{|l|}{ Tumor types (Grading) } \\
\hline Pilocytic astrocytoma (I) & $2(4.3 \%)$ & 0 & 0 & $2(100 \%)$ \\
\hline Astrocytoma (II) & $7(14.9 \%)$ & $1(14.3 \%)$ & 0 & $6(85.7 \%)$ \\
\hline Anaplastic astrocytoma (III) & $9(19.1 \%)$ & $1(11.1 \%)$ & 0 & $8(88.9 \%)$ \\
\hline Primary GBM (IV) & $24(51 \%)$ & 0 & $1(4.2 \%)$ & $23(95.8 \%)$ \\
\hline Secondary GBM (IV) & $1(2.1 \%)$ & 0 & 0 & $1(100 \%)$ \\
\hline Oligodendroglioma (II) & $1(2.1 \%)$ & 0 & 0 & $1(100 \%)$ \\
\hline Anaplastic oligodendroglioma (III) & $1(2.1 \%)$ & 0 & 0 & $1(100 \%)$ \\
\hline Anaplastic oligoastrocytoma (III) & $1(2.1 \%)$ & 0 & 0 & $1(100 \%)$ \\
\hline Anaplastic ependymomas (III) & $1(2.1 \%)$ & 0 & 0 & $1(100 \%)$ \\
\hline Percentage of IDH1 mutations in samples & $3(6.4 \%)$ & & & \\
\hline
\end{tabular}

Table 2: Association between the IDH1 gene status with age and gender among 47 glioma samples.

\begin{tabular}{|c|c|c|c|c|}
\hline \multirow[b]{2}{*}{ Characteristic } & \multirow[b]{2}{*}{ No. of samples } & \multicolumn{3}{|c|}{ IDH1 status } \\
\hline & & IDH1 R132H & IDH1 R132L & $\begin{array}{c}\text { IDH1 } \\
\text { wild-type }\end{array}$ \\
\hline No of samples & 47 & $2(4.3 \%)$ & $1(2.1 \%)$ & $44(93.6 \%)$ \\
\hline Median age & & 26.5 & 38 & 44 \\
\hline \multicolumn{5}{|l|}{ Gender } \\
\hline Male & $31(66 \%)$ & $2(6.5 \%)$ & & $29(93.5 \%)$ \\
\hline Female & $16(34 \%)$ & 0 & $1(6.3 \%)$ & $15(93.7 \%)$ \\
\hline Percentage of IDH1 mutations in samples & $3(6.4 \%)$ & & & \\
\hline
\end{tabular}


PCR-RFLP method was only able to detect IDH1 R132H, R132C, R132L, R132G, and R132S but not novel mutations. However, it was sufficient to confirm the presence of IDH1 $\mathrm{R} 132 \mathrm{H}$ and IDH1 R132L mutations in our samples. The presence of two peaks at the same position was expected as IDH1 mutations are somatic heterozygous mutations (20). Approximately $6.4 \%$ IDH1 mutations consisting of two IDH1 R132H and one IDH1 R132L were found in 47 glioma samples.

In line with previous IDH1 studies in gliomas, IDH1 R132H was the most common mutation, $4.3 \%$ in our glioma samples. It was found in grade II astrocytoma and grade III anaplastic astrocytoma. Although it was the most common mutation in our sample, it was relatively low compared to other IDH1 R132H studies in gliomas, $15 \%$ (21), $23.4 \%$ (22), 30.7\% (23), $31.6 \%$ (24), $43.6 \%$ (25), 48\% (4), $54.7 \%$ (18), 82\% (26) and $85.4 \%$ (27).

IDH1 R132L mutation was found in $2.1 \%$ of our sample. The mutation was found in primary GBM, therefore the glioma was suspected to be secondary GBM but had escaped early low grade glioma diagnosis. Various studies agreed that this mutation existed in very a low percentages, $0.6 \%(24), 0.8 \%(25)$, $0.9 \%(21), 2 \%(26)$ and $8 \%(4)$.

There were various factors contributed to the low percentages of IDH1 mutation in our samples. One of the factors was small sample size compared to other glioma studies ranging from 50 to 1010 gliomas $(4,12,18)$. Another factor was the sensitivity of DNA sequencing method. DNA sequencing requires a minimum of $20-25 \%$ mutant allele load in the background of normal genomic DNA to be detected (28).

Gliomas are invasive tumors which have no distinct border between tumor cells and normal brain cells (29). Therefore, glioma biopsy samples are commonly contaminated with the normal brain cells which dilute the true tumor genomic DNA that leads to false negative results. This can be seen from the DNA electropherogram results where the spectrum of Guanine nucleotide (IDH1 wild-type) was higher than the mutant nucleotide, Adenine (R132H) and Thymine (R132L) at 395th position. This leads to the failure of detecting IDH1 mutations in the samples. Laser microdissection or cell sorting can be used to harvest tumor cells from the heterogenous glioma samples. However, these equipment are not available in common laboratory setting and not suitable for routine clinical testing due to high cost and time-consuming.

Selection bias may also attributed to the low percentages of IDH1 mutations as well. This was because only available FFPE glioma blocks were included in the study. More than $50 \%$ of our samples were consists of pilocytic astrocytomas, primary GBMs, and ependymoma which were reported rarely to harbor IDH1 mutations (30). Our GBMs samples were comparable with Ohgaki et al., 2004 (31) as we also found secondary GBM was rare, $1 / 25$ (4\%) compared to primary GBMs, 24/25 (96\%). Our glioma samples were from Malay ethnic only which may be one of the factors as no similar study had been done on other ethnics in Malaysia.

We did observed that the IDH1 mutant glioma patients were generally younger compared to the IDH1 wild-type glioma patients but the association remain inconclusive as the percentage of IDH1 mutations in our sample was too small for validation. However, IDH1 mutation were widely agreed to be found in younger glioma patients compared to IDH1 wild-type which often found in older patients (32).

\section{Conclusions}

We discovered common IDH1 R132H and rare IDH1 R132L mutations in our glioma samples. Our study showed that DNA sequencing method for IDH1 mutation detection in glioma is insufficient due to the heterogeneous nature of the glioma samples and low sensitivity level. PCR-RFLP method was capable to screen IDH1 mutations but cannot identify the actual IDH1 mutations. Hence, combination of DNA sequencing and PCRRFLP method in heterogeneous glioma samples can be applied to avoid false negative result.

\section{Acknowledgments}

WCG conducted the experiments, collected the data and contributed the writing of the manuscript. BI and RK analyzed the study results and contributed the revision of manuscript. SS assisted in genetic analysis data and contributed the writing of the manuscript. $\mathrm{HJ}$ reviewed and confirmed the stage and types of brain tumors. FA designed, directed the study and reviewed the study results. The author thanks Dr. Siti Azrin for her expertise in biostatistics.

This work was supported by the USM Short Term grant (304/ PPSP/6315115) and the USM Fellowship provided by Universiti Sains Malaysia.

\section{Conflict of Interest}

The authors declared they do not have anything to disclose regarding conflict of interest with respect to this manuscript.

\section{References}

1. Donovan LE, Lassman AB. Chemotherapy Treatment and Trials in Low - Grade Gliomas. Neurosurgery Clinics of North America. 2019; 30(1):103-109.

2. Li S, Chou AP, Chen W, et al. Overexpression of isocitrate dehydrogenase mutant proteins renders glioma cells more sensitive to radiation. Neuro Oncology. 2013; 15(1):57-68.

3. Goh CH, Lu YY, Lau BL, et al. Brain and spinal tumour. Medical Journal Malaysia. 2014; 69(6):261-267.

4. Agarwal S, Sharma MC, Jha P, et al. Comparative study of IDH1 mutations in gliomas by immunohistochemistry and DNA sequencing. Neuro Oncology. 2013; 15(6):718-726.

5. Parsons DW, Jones S, Zhang X, et al. An integrated genomic analysis of human glioblastoma multiforme. Science. 2008; 321(5897):1807-1812.

6. Losman JA, Jr WGK. What a difference a hydroxyl makes : mutant IDH , ( R ) -2-hydroxyglutarate , and cancer. Genes Development. 2013; 27(8):836-852.

7. Liu A, Hou C, Chen H, Zong X, Zong P. Genetics and Epigenetics of Glioblastoma: Applications and Overall Incidence of IDH1 Mutation. Frontier Oncology. 2016; 6(16):1-9.

8. Ohgaki H, Kleihues P. The Definition of Primary and Secondary Glioblastoma. Clinical Cancer Research. 2013; 19(4):764-773.

9. Hartmann C, Hentschel B, Wick W, et al. Patients with IDH1 wild type anaplastic astrocytomas exhibit worse 
prognosis than IDH1-mutated glioblastomas, and IDH1 mutation status accounts for the unfavorable prognostic effect of higher age: Implications for classification of gliomas. Acta Neuropathologica. 2010; 120(6):707-718.

10. Dang L, Yen K, Attar EC. IDH mutations in cancer and progress toward development of targeted therapeutics. Annals Oncology. 2016; 27(4):599-608.

11. Highsmith W. Electrophoretic Methods for Mutation Detection and DNA Sequencing.2nd ed. Humana Press;2006.

12. Hartmann C, Meyer J, Balss J, et al. Type and frequency of IDH1 and IDH2 mutations are related to astrocytic and oligodendroglial differentiation and age: A study of 1,010 diffuse gliomas. Acta Neuropathologica. 2009; 118(4):469-474.

13. Balss J, Meyer J, Mueller W, Korshunov A, Hartmann C, von Deimling A. Analysis of the IDH1 codon 132 mutation in brain tumors. Acta Neuropathologica. 2008;116(6):597-602.

14. Bleeker FE, Atai NA, Lamba S, et al. The prognostic IDH1R132 mutation is associated with reduced NAD$\mathrm{P}+$-dependent IDH activity in glioblastoma. Acta Neuropathologica. 2010; 119(4):487-494.

15. Aziz A, Yusoff M, Zulfakhar FN, Sul'ain MD, Idris Z, Abdullah JM. Association of The IDH1 C . 395G > A ( R132H ) Mutation with Histological Type in Malay Brain Tumors. Asian Pacific J Cancer Prevention. 2016; 17(12):51955201.

16. David NL, Perry A, Reifenberger G, et al. The 2016 World Health Organization Classification of Tumors of the Central Nervous System: a summary. Acta Neuropathologica. 2016; 131(6):803-820.

17. Meyer J, Pusch S, Balss J, et al. PCR- and restriction endonuclease-based detection of IDH1 mutations. Brain Pathology. 2010; 20(2):298-300.

18. Preusser M, Wohrer A, Stary S, Hoftberger R, Streubel $B$, Hainfellner JA. Value and limitations of immunohistochemistry and gene sequencing for detection of the IDH1-R132H mutation in diffuse glioma biopsy specimens. J Neuropathol Exp Neurol.2011;70(8):715-723.

19. Van Den Bent MJ, Hartmann C, Preusser M, et al. Interlaboratory comparison of IDH mutation detection. $\mathrm{J}$ Neurooncology. 2013; 112(2):173-178.

20. Luchman HA, Stechishin OD, Dang NH, et al. An in vivo patient-derived model of endogenous IDH1 -mutant glioma. Neuro Oncology. 2012; 14(2):184-191.

21. Bleeker FE, Lamba S, Leenstra S, et al. IDH1 mutations at residue p.R132 (IDH1R132) occur frequently in highgrade gliomas but not in other solid tumors. Human Mutation. 2009; 30(1):7-11.

22. Setty P, Hammes J, Rothamel T, et al. A pyrosequencing-based assay for the rapid detection of IDH1 mutations in clinical samples. J Mol Diagnostic. 2010; 12(6):750-756.

23. Wang J, Zhao Y-Y, Li J-F, et al. IDH1 mutation detection by droplet digital PCR in glioma. Oncotarget. 2015;6(37):
39651-39660.

24. Gupta R, Flanagan S, Li CC, et al. Expanding the spectrum of IDH1 mutations in gliomas. Modern Pathology. 2013; 26(5):619-625.

25. Catteau A, Girardi H, Monville F, et al. A new sensitive PCR assay for one-step detection of 12 IDH1/2 mutations in glioma. Acta Neuropathol Communications. 2014; 2(1):1-12.

26. Horbinski C, Kelly L, Nikiforov YE, Durso MB, Nikiforova MN. Detection of IDH1 and IDH2 mutations by fluorescence melting curve analysis as a diagnostic tool for brain biopsies. J Mol Diagnostic. 2010; 12(4): 487-492.

27. Loussouarn D, Le Loupp AG, Frenel JS, et al. Comparison of immunohistochemistry, DNA sequencing and allele-specific PCR for the detection of IDH1 mutations in gliomas. Int J Oncology. 2012; 40(6):2058-2062.

28. Boisselier $B$, Marie $Y$, Labussière $M$, et al. COLD PCR HRM: A highly sensitive detection method for IDH1 mutations. Human Mutation. 2010; 31(12):1360-1365.

29. Furnari FB, Fenton T, Bachoo RM, et al. Malignant astrocytic glioma: Genetics, biology, and paths to treatment. Genes Development. 2007; 21(21):2683-2710.

30. Ichimura K, Pearson DM, Kocialkowski S, et al. IDH1 mutations are present in the majority of common adult gliomas but rare in primary glioblastomas. Neuro Oncology. 2009; 11(4):341-347.

31. Ohgaki $H$, Dessen $P$, Jourde B, et al. Genetic Pathways to Glioblastoma : A Population-Based Study CANCER RESEARCH. 2004; 64(19): 6892-6899.

32. Bujko M, Kober P, Matyja E, et al. Prognostic value of IDH1 mutations identified with PCR-RFLP assay in glioblastoma patients. Mol Diagnosis Therapy. 2010; 14(3):163-169. 